\title{
Basis set superposition error along the free-energy surface of the water dimer. SUPPLEMENTARY MATERIAL
}

\author{
Jens Thar $^{\dagger}$, Rainer Hovorka ${ }^{\ddagger}$ and Barbara Kirchner ${ }^{\dagger 1}$ \\ † Lehrstuhl für Theoretische Chemie, Wilhelm-Ostwald Institut für Physikalische und \\ Theoretische Chemie, \\ Universität Leipzig, Linnestr. 2, D-04103 Leipzig, Germany \\ ‡ Organische Chemie, Kekulé Institut für Organische Chemie und Biochemie, \\ Universität Bonn, Gerhard-Domagk-Str. 1, D-53121 Bonn, Germany
}

\footnotetext{
${ }^{1}$ Corresponding author: B. Kirchner; email: bkirchner@uni-leipzig.de
} 


\section{Introduction}

It also can be recognized from Fig. 1 in the artcile that the size of the BSSE strongly depends on the geometry of the water dimer. It would therefore be difficult to work with Gaussian basis sets in simulations. So far no detailed study was published concerning the basis set superposition error in simulations. The reliability of (Gaussian-type) basis sets is usually only tested for static quantum chemical calculations and in the majority of cases for intramolecular properties like bond lengths; see, e.g., Refs. ${ }^{1-3}$ However, the very recent article of Haynes and coworkers is interesting in our context, because the authors showed that the BSSE is eliminated by optimizing the local orbitals in situ using a basis set that is similar to a PWBS. ${ }^{4}$

In this supplemnetary material part we compare static calculations of the water dimer from a plane wave basis set with calculations from Gaussian basis sets. We investigate the two minimum structures shown in Fig. 1.
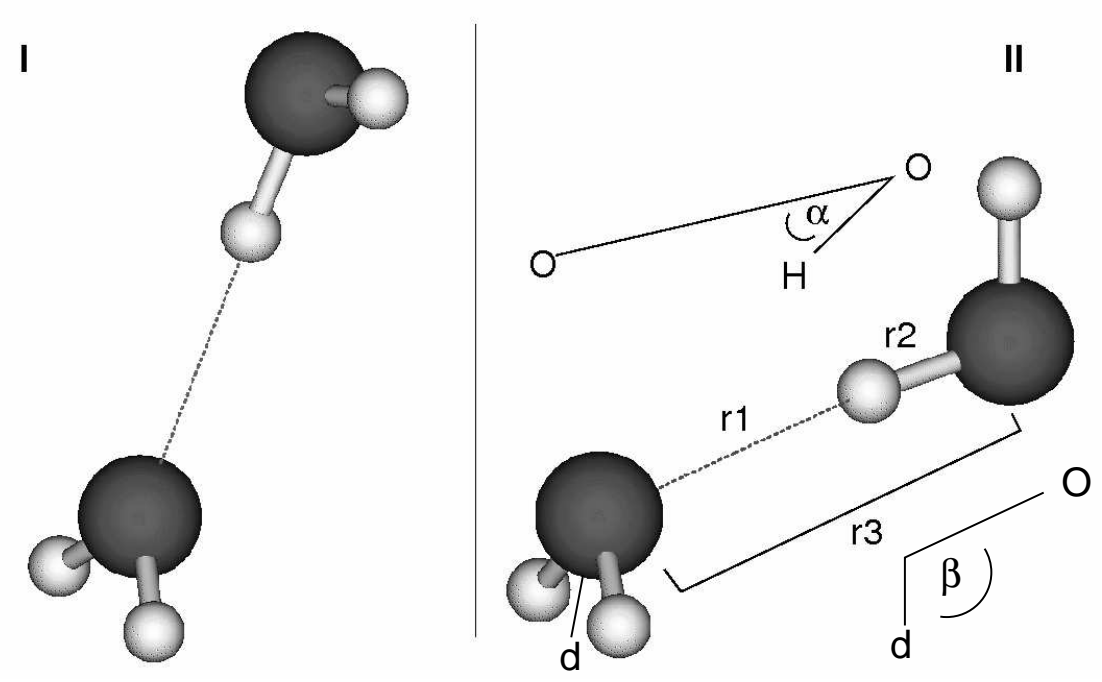

Figure 1: Two different water-dimer conformations. Left: Conformation I is a flat structure; Right: Conformation II indicates the experimental minimum structure. Dark spheres mark oxygen atoms. 


\section{Technical Part}

\subsection{Plane waves}

Plane waves,

$$
P W \sim \exp [i \mathbf{G} \cdot \mathbf{r}]
$$

build a complete and orthonormal basis with an inherent periodicity, where $\mathbf{G}$ is the reciprocal space vector and $\mathbf{r}$ is the electronic coordinate. ${ }^{5}$ The inherent periodicity is very important for the application of simulations. Periodic boundary conditions are a necessary ingredient to avoid surface effects and to model bulk systems. ${ }^{6}$ If a simulation takes place in a cubic box this box is replicated throughout space to form an infinite lattice.

A periodic function can be expanded in the basis of the form given in Eq. (1):

$$
\psi(\mathbf{r})=\psi(\mathbf{r}+\mathbf{L}) \sim \sum_{\mathbf{G}} \psi(\mathbf{G}) \exp [i \mathbf{G} \cdot \mathbf{r}] .
$$

The functions $\psi(\mathbf{r})$ and $\psi(\mathbf{G})$ are related by a three-dimensional Fourier transform. The direct lattice vectors $\mathbf{L}$ connect equivalent points in different cells. The Kohn-Sham orbitals can be written in Bloch form,

$$
\phi_{i}(\mathbf{r})=\phi_{i}(\mathbf{r}, \mathbf{k})=\exp [i \mathbf{k} \cdot \mathbf{r}] u_{i}(\mathbf{r}, \mathbf{k}),
$$

where $\mathbf{k}$ is a vector in the first Brillouin zone. The functions $u_{i}(\mathbf{r}, \mathbf{k})$ hold the periodicity of the direct lattice,

$$
u_{i}(\mathbf{r}, \mathbf{k})=u_{i}(\mathbf{r}+\mathbf{L}, \mathbf{k}) .
$$

The index $i$ runs over all one-particle states and these are associated with an occupation. Expanding now the periodic functions $u_{i}(\mathbf{r}, \mathbf{k})$ in the plane wave basis leads to

$$
u_{i}(\mathbf{r}, \mathbf{k}) \sim \sum_{\mathbf{G}} c_{i}(\mathbf{G}, \mathbf{k}) \exp [i \mathbf{G} \cdot \mathbf{r}]
$$

and the Kohn-Sham orbitals are now given as

$$
\phi_{i}(\mathbf{r}, \mathbf{k}) \sim \sum_{\mathbf{G}} c_{i}(\mathbf{G}, \mathbf{k}) \exp [i(\mathbf{G}+\mathbf{k}) \cdot \mathbf{r}]
$$

In the actual calculations when the density is used the integration over the Brillouin zone has to be approximated by a finite sum over special k-points. Furthermore, the infinite sum over $\mathbf{G}$ vectors and cells (stems from the pre-factor which is omitted here for brevity) has to be truncated. Since the Kohn-Sham potential converges rapidly with increasing modulus of $\mathbf{G}$, such $\mathbf{G}$ vectors with a kinetic energy higher than a given maximum cutoff $E_{\text {cut }}$ are neglected,

$$
\frac{1}{2}|\mathbf{G}|^{2} \leq E_{\text {cut }}
$$




\subsection{Computational details}

\subsubsection{Static quantum chemical calculations}

All standard quantum chemical results were obtained from density functional theory (DFT) employing the restricted Kohn-Sham ansatz. Those using a GBS were performed with the program TURBOMOLE ${ }^{7}$ and those using a PWBS were performed with the CPMD program package. $^{8}$ For comparison we also carried out optimizations and single-point calculations on Turbomole-geometries of corresponding basis set with the $\mathrm{CP} 2 \mathrm{~K}$ code. ${ }^{9}$ The different density functional theory calculations were performed with the gradient corrected functionals

- $\mathrm{BLYP},{ }^{10,11} \mathrm{BP} 86,{ }^{10,12}$ and $\mathrm{PBE}^{13}$

as well as hybrid functionals

- B3LYP ${ }^{14,15}$ and PBE0. ${ }^{16}$

For the gradient-corrected functionals the resolution of the identity or density fitting technique was applied. ${ }^{17,18}$ Please note that the hybrid functionals of CPMD and TURBOMOLE are not directly comparable. The first CPMD simulation employing hybrid functionals in the plane wave approach was carried out in the Hutter group. ${ }^{19}$

The quality of the plane wave basis set was determined by the energy cutoff $E_{\text {cut }}$ which we chose to be 20, 50, 70, 90, 110, 150 and 300 Ryd.

The Gaussian basis sets were selected from

- Ahlrichs' basis sets SVP, TZVP, TZVPP, and TZVPPP, ${ }^{20}$ and

- Dunning's basis sets cc-pVDZ, cc-pVTZ, cc-pVQZ, cc-pV5Z, ${ }^{21}$

Table 1 lists some of the basis sets including Pople's basis sets $3-21 \mathrm{G}$ and $6-31 \mathrm{G}^{\star \star} .{ }^{22}$ and their contractions for comparison. 
Table 1: Contraction schemes of some of the basis sets for $\mathrm{H}$ and $\mathrm{O}$.

\begin{tabular}{lcc}
\hline \hline basis & $\mathrm{H}$ & $\mathrm{O}$ \\
\hline $3-21 \mathrm{G}$ & $(5 \mathrm{~s}) /[2 \mathrm{~s}]$ & $(6 \mathrm{~s} 3 \mathrm{p}) /[3 \mathrm{~s} 2 \mathrm{p}]$ \\
$6-31 \mathrm{G} \star \star$ & $(10 \mathrm{~s} 3 \mathrm{p}) /[3 \mathrm{~s} 1 \mathrm{p}]$ & $(10 \mathrm{~s} 4 \mathrm{p} 1 \mathrm{~d}) /[3 \mathrm{~s} 2 \mathrm{p} 1 \mathrm{~d}]$ \\
SVP & $(7 \mathrm{~s}) /[3 \mathrm{~s}]$ & $(7 \mathrm{~s} 4 \mathrm{p} 1 \mathrm{~d}) /[3 \mathrm{~s} 2 \mathrm{p} 1 \mathrm{~d}]$ \\
TZVP & $(10 \mathrm{~s}) /[4 \mathrm{~s}]$ & $(11 \mathrm{~s} 6 \mathrm{p} 1 \mathrm{~d}) /[5 \mathrm{~s} 3 \mathrm{p} 1 \mathrm{~d}]$ \\
TZVPP & $(11 \mathrm{~s} 4 \mathrm{p}) /[5 \mathrm{~s} 1 \mathrm{p}]$ & $(11 \mathrm{~s} 6 \mathrm{p} 2 \mathrm{~d} 1 \mathrm{f}) /[5 \mathrm{~s} 3 \mathrm{p} 2 \mathrm{~d} 1 \mathrm{f}]$ \\
cc-pVDZ & $(4 \mathrm{~s} 1 \mathrm{p}) /[2 \mathrm{~s} 1 \mathrm{p}]$ & $(9 \mathrm{~s} 4 \mathrm{p} 1 \mathrm{~d}) /[3 \mathrm{~s} 2 \mathrm{p} 1 \mathrm{~d}]$ \\
cc-pVTZ & $(5 \mathrm{~s} 2 \mathrm{p} 1 \mathrm{~d}) /[3 \mathrm{~s} 2 \mathrm{p} 1 \mathrm{~d}]$ & $(10 \mathrm{~s} 5 \mathrm{p} 2 \mathrm{~d} 1 \mathrm{f}) /[4 \mathrm{~s} 3 \mathrm{p} 2 \mathrm{~d} 1 \mathrm{f}]$ \\
cc-pVQZ & $(6 \mathrm{~s} 3 \mathrm{p} 2 \mathrm{~d} 1 \mathrm{f}) /[4 \mathrm{~s} 3 \mathrm{p} 2 \mathrm{~d} 1 \mathrm{f}]$ & $(12 \mathrm{~s} 6 \mathrm{p} 3 \mathrm{~d} 2 \mathrm{f} 1 \mathrm{~g}) /[5 \mathrm{~s} 4 \mathrm{p} 3 \mathrm{~d} 2 \mathrm{f} 1 \mathrm{~g}]$ \\
cc-pV5Z & $(8 \mathrm{~s} 4 \mathrm{p} 3 \mathrm{~d} 2 \mathrm{f} 1 \mathrm{~g}) /[5 \mathrm{~s} 4 \mathrm{p} 3 \mathrm{~d} 2 \mathrm{f} 1 \mathrm{~g}]$ & $(14 \mathrm{~s} 8 \mathrm{p} 4 \mathrm{~d} 3 \mathrm{f} 2 \mathrm{~g} 1 \mathrm{~h}) /[6 \mathrm{~s} 5 \mathrm{p} 4 \mathrm{~d} 3 \mathrm{f} 2 \mathrm{~g}]$ \\
\hline \hline
\end{tabular}

It should be stressed here that the TZVP basis set is superior to the $6-31 \mathrm{G}^{* *}$ for the oxygen atom. From the quadrupole zeta basis set on the Dunning basis consists of more functions than the chosen Ahlrichs basis set. All structures were optimized until the SCF convergence criterion $10^{-6}$ has been reached in the selfconsistent field iterations. The threshold for the norm of the gradient was set to $10^{-4}$. For CP2K the SCF convergence criterion is set to $10^{-6}$, the cutoff to 320 Ryd, the relative cutoff is 40 Ryd and box size is $1200 \mathrm{pm}$.

All PWBS calculations were performed with the CPMD code. ${ }^{8}$ The convergence criteria for the plane wave basis set calculations were kept at the standard values. The maximum values for the largest element of the wavefunction gradient are $10^{-5}$ and $5 \cdot 10^{-4}$ for the ions. The cutoff radii for each functional are given in the results section. We used the norm-conserving Troullier-Martins type pseudopotentials. In the pseudopotential generation, the cutoff for O was set to 1.05 a.u. (BLYP), 1.4 a.u. (BP86), and 1.12 a.u. (PBE) for all angular momenta. The pseudopotentials were taken form the library of Boero. ${ }^{8}$ The size of the box for the plane wave calculations was set to $1323 \mathrm{pm}$.

To enable the study of isolated systems, the inherent periodicity of the plane-wave calculations for CPMD as well as $\mathrm{CP} 2 \mathrm{~K}$ was circumvented for by applying an appropriate Poisson solver designed for nonperiodic boundary conditions. ${ }^{23}$

\section{Results}

\subsection{Static Calculations}

Table 2 shows total energies from optimizations of both water dimer structures for different functionals and different energy cutoffs of the plane wave basis set. Fig. 2 depicts a graphical representation of the convergence behavior. For the BLYP functional the basis set limit as shown previously by Lee and Tuckerman ${ }^{24}$ of 300 Ryd deviates from the value at 150 Ryd only 
by approximately $5 \mathrm{~kJ} / \mathrm{mol}$. We see that independent of the functional and of the water dimer structures, the calculations are converged within the first digit before the decimal point for $E_{c u t}=50 \mathrm{Ryd}$, with respect to the reference calculation. The difference between 50 Ryd and 70 Ryd is thus in the range of $800 \mathrm{~kJ} / \mathrm{mol}$, except for the BP86 functional which only shows a difference of $250 \mathrm{~kJ} / \mathrm{mol}$. Using the standard cutoff of $70 \mathrm{Ryd}$, the energies converge within the first digit after the decimal point. This means that the error lowers to about $180 \mathrm{~kJ} / \mathrm{mol}$. Choosing the cutoff of 90 Ryd improves the convergence behavior to 0.01 hartree, i.e. an error of about $25 \mathrm{~kJ} / \mathrm{mol}$. Therefore we also recommend using a cutoff of 90 Ryd in calculations with systems containing water molecules if computer time is available as it is in general recommended but currently seldomly used. For a qualitative discussion a cutoff of 70 Ryd might be sufficient to capture all important chemical effects. We will come back to this point when discussing binding energies which correspond to chemical effects.

Table 2: Total energies $E_{\text {tot }}$ in hartree from plane wave optimization. Cutoff energy $E_{\text {cut }}$ in Ryd. First block: Water dimer structure I; Second block: Water dimer structure II.

\begin{tabular}{lccccc}
\hline \hline & & \multicolumn{4}{c}{$E_{\text {tot }}(\mathbf{I})$} \\
\cline { 3 - 5 }$E_{\text {cut }}$ & BP86 & BLYP & B3LYP & PBE & PBE0 \\
\hline 20 & -32.48863 & -32.07206 & -32.03749 & -32.09725 & -32.10208 \\
50 & -34.33881 & -34.05670 & -34.01019 & -34.04635 & -34.04413 \\
70 & -34.43300 & -34.33538 & -34.28646 & -34.34203 & -34.33711 \\
90 & -34.44052 & -34.39582 & -34.34721 & -34.41287 & -34.40825 \\
110 & -34.44359 & -34.40496 & -34.35668 & -34.42506 & -34.42505 \\
150 & -34.44636 & -34.40842 & -34.36009 & -34.43462 & -34.42878 \\
300 & - & -34.41029 & - & - & - \\
\hline & & & $E_{\text {tot }}(\mathbf{I I})$ & & \\
$E_{\text {cut }}$ & BP86 & BLYP & B3LYP & PBE & PBE0 \\
\hline 20 & -32.49419 & -32.07742 & -32.04218 & -32.10284 & -32.10705 \\
50 & -34.34032 & -34.05818 & -34.01118 & -34.04809 & -34.04548 \\
70 & -34.43456 & -34.33672 & -34.28766 & -34.34359 & -34.33843 \\
90 & -34.44206 & -34.39708 & -34.34836 & -34.41437 & -34.40956 \\
110 & -34.44513 & -34.40621 & -34.35782 & -34.42654 & -34.42210 \\
150 & -34.44787 & -34.40960 & -34.36125 & -34.43023 & -34.42583 \\
300 & - & -34.41153 & - & - & - \\
\hline \hline
\end{tabular}




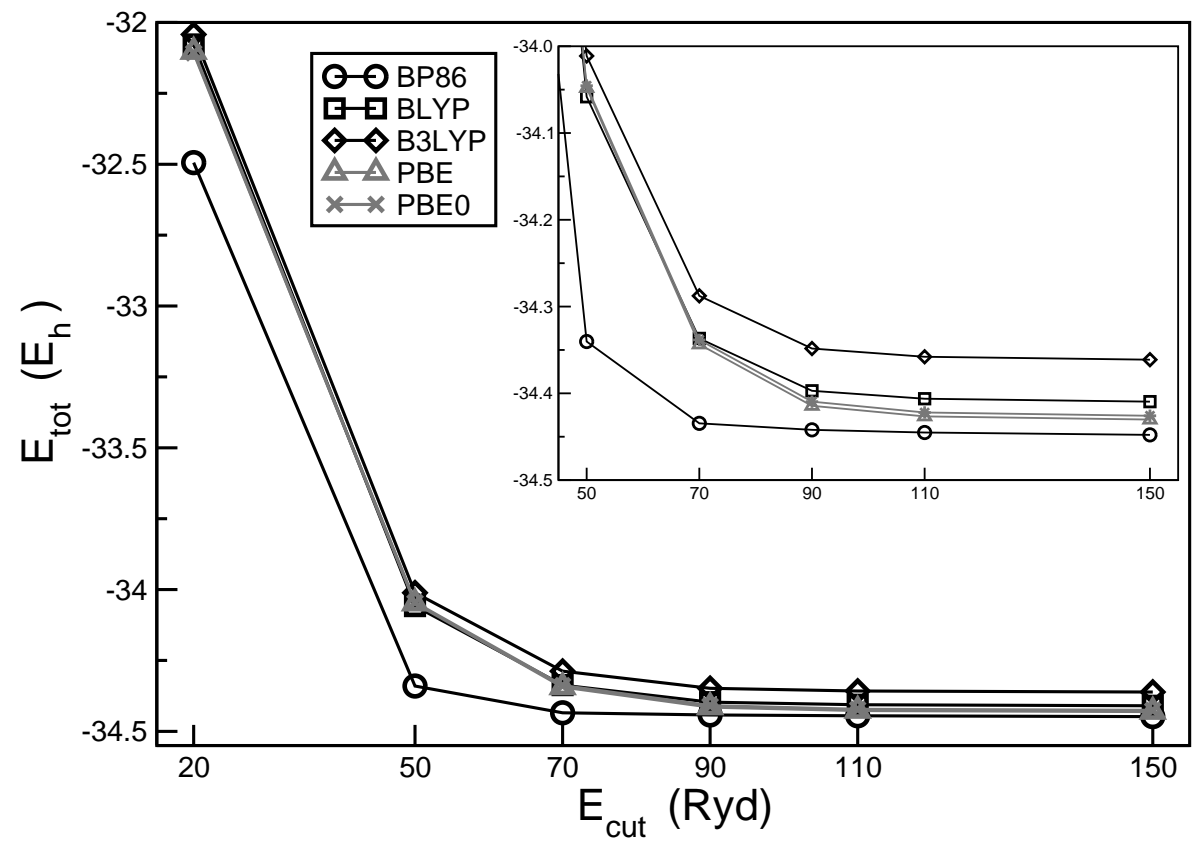

Figure 2: The convergence behavior of the total energy $E_{\text {tot }}$ obtained from PWBS/DFT calculations at structure II employing different functionals as a function of the energy cutoff $E_{c u t}$ in Ryd.

Fig. 2 reflects the behavior discussed. Since the convergence behavior is the same for structure I and II we show only the behavior of structure II. Subtle changes are better gathered in Fig. 2 than in table 2. The behavior for all functionals is similar, except that the BP86 curve starts at lower energies and converges faster. 
Table 3: Interaction energy $E_{\mathrm{I}}$ in $\mathrm{kJ} / \mathrm{mol}$ from plane wave calculations. Cutoff energy $E_{\text {cut }}$ in Ryd. First block: At water dimer structure I; Second block: At water dimer structure II.

\begin{tabular}{lrrrrr}
\hline \hline & & \multicolumn{5}{c}{$E_{I}(\mathbf{I})$} & \\
\cline { 3 - 5 }$E_{\text {cut }}$ & BP86 & BLYP & B3LYP & PBE & PBE0 \\
\hline 20 & -2.79 & -1.37 & -2.34 & -6.48 & -4.43 \\
50 & -14.08 & -12.65 & -14.07 & -15.79 & -15.78 \\
70 & -14.69 & -14.51 & -15.97 & -17.88 & -17.86 \\
90 & -14.44 & -14.49 & -15.97 & -17.93 & -17.88 \\
110 & -14.44 & -14.39 & -15.88 & -17.89 & -17.84 \\
150 & -14.41 & -14.34 & -15.81 & -17.94 & -17.83 \\
300 & - & -14.25 & - & - & - \\
\hline & & & $E_{I}(\mathbf{I I})$ & & \\
$E_{\text {cut }}$ & BP86 & BLYP & B3LYP & PBE & PBE0 \\
\hline 20 & -18.37 & -16.63 & -15.71 & -22.15 & -18.53 \\
50 & -17.89 & -16.52 & -16.70 & -20.42 & -19.39 \\
70 & -18.77 & -18.03 & -19.18 & -22.01 & -21.34 \\
90 & -18.57 & -17.76 & -18.96 & -21.87 & -21.27 \\
110 & -18.52 & -17.64 & -18.89 & -21.78 & -21.23 \\
150 & -18.40 & -17.44 & -18.84 & -21.66 & -21.14 \\
300 & - & -17.50 & - & - & - \\
\hline \hline
\end{tabular}

The interaction energies are listed in table 3 for all functionals and energy cutoffs. For every choice of cutoff and functional structure $\mathbf{I}$ is more weakly interacting than structure II. The difference in interaction energies between the two structures converges to approximately 3 $\mathrm{kJ} / \mathrm{mol}$ independently of the particular functional. We also see the usual functional dependencies, for example both PBE functionals give stronger binding energies than all other functionals and BP86 yields a higher binding energy than BLYP. Table 3 shows that obviously more than one structure should be investigated, because for all values of $E_{\text {cut }}$ structure II yields a reasonable interaction energy. Inspecting the results for structure I we realize that a cutoff energy of 20 Ryd is leading to absurd results, while cutoff 20 Ryd yields good results for structure II. It has been long recognized that a too small cutoff leads to unreliable energetic results. In our considerations this is due to the oxygen atom, because this electronegative element of the first row needs a higher cutoff, due to its compact valence shell.

From cutoff 70 Ryd and even 50 Ryd on we obtain comparable interaction energies for all chosen cutoffs of a particular functional. This is the reason why we expect cutoff 70 Ryd to capture important "chemical effects" despite the fact that for all chosen cutoffs and some functionals the results are not within "chemical accuracy" which is simply due to density functional theory and has nothing to do with basis set convergence. 
Similar trends were found by the Hutter group in their study of hybrid functionals applied to water simulations. ${ }^{19}$ The authors calculated interaction energies of $18.16 \mathrm{~kJ} / \mathrm{mol}$ for BLYP, $19.37 \mathrm{~kJ} / \mathrm{mol}$ for B3LYP, $19.08 \mathrm{~kJ} / \mathrm{mol}$ for PBE, and $19.96 \mathrm{~kJ} / \mathrm{mol}$ for PBE0. The difference of the latter two values to our values are about $3 \mathrm{~kJ} / \mathrm{mol}$ and might be attributed to the choice of the pseudopotentials.

Table 4: Total energies $E_{t o t}$ in hartree optimized with GBS for structure II. The GBS were abbreviated for clarity: TZV2P = TZVPP; TZV3P = TZVPPP; $\mathrm{C}=\mathrm{cc}-\mathrm{p}$

\begin{tabular}{lccccc}
\hline \hline & & \multicolumn{3}{c}{$E_{\text {tot }}(\mathbf{I I})$} & \multirow{2}{*}{ PBE0 } \\
\cline { 3 - 5 } basis set & BP86 & BLYP & B3LYP & PBE & \\
\hline SVP & -152.74006 & -152.68965 & -152.65561 & -152.56136 & -152.56598 \\
TZVP & -152.93888 & -152.89529 & -152.85656 & -152.75913 & -152.75962 \\
TZV2P & -152.94962 & -152.90597 & -152.86743 & -152.76985 & -152.77047 \\
TZV3P & -152.95154 & -152.90814 & -152.86941 & -152.77182 & -152.77223 \\
& & & & & \\
CVDZ & -152.86397 & -152.81152 & -152.78010 & -152.68310 & -152.69105 \\
CVTZ & -152.93732 & -152.89264 & -152.85513 & -152.75708 & -152.75876 \\
CVQZ & -152.95584 & -152.91274 & -152.87353 & -152.77618 & -152.77602 \\
CV5Z & -152.96255 & -152.92056 & -152.88011 & -152.78343 & -152.78193 \\
Limit & -152.964 & -152.922 & -152.881 & -152.785 & -152.783 \\
\hline \hline
\end{tabular}

Table 4 shows the convergence behavior of the DFT calculations employing GBSs. Changing from SVP to TZVP leads to a gain in accuracy at the first digit after the decimal point. The next step from TZVP to TZVPP basis set cuts the remaining error in half while a change to the TZVPPP basis set yields only minor improvements. The chosen Dunning basis sets (cc-pVXZ) at the double zeta quality are converged within the first digit before the decimal point. From the triple zeta basis set on, the energies are converged within the first digit after the decimal point. If we try to compare the convergence behavior we could compare the SVP basis set to a cutoff $E_{\text {cut }}$ of 50 Ryd. The TZVP basis set convergence may be compared to $E_{\text {cut }}=70$ Ryd and the TZVPP to $E_{\text {cut }}=90$ Ryd. 
Table 5: BSSE in $\mathrm{kJ} / \mathrm{mol}$ from GBS calculations at conformation II. The GBS were abbreviated for consistency: TZV2P $=$ TZVPP; TZV3P $=$ TZVPPP; $\mathrm{C}=$ cc-p The last two lines show values obtained with the GAPW code CP2K.

\begin{tabular}{lccccc}
\hline \hline & & \multicolumn{3}{c}{ BSSE(II) } & \\
\cline { 3 - 5 } basis & BP86 & BLYP & B3LYP & PBE & PBE0 \\
\hline SVP & -17.48 & -29.34 & -16.56 & -27.49 & -13.94 \\
TZVP & -2.95 & -3.25 & -2.67 & -3.36 & -2.58 \\
TZV2P & -2.73 & -3.42 & -2.60 & -3.34 & -2.40 \\
TZV3P & -2.81 & -3.57 & -2.68 & -3.49 & -2.41 \\
& & & & & \\
CVDZ & -17.76 & -20.55 & -16.53 & -19.59 & -14.32 \\
CVTZ & -6.94 & -8.45 & -6.72 & -8.09 & -5.77 \\
CVQZ & -3.61 & -4.27 & -3.25 & -4.49 & -2.90 \\
CV5Z & -1.19 & -1.42 & -1.01 & -1.39 & -0.89 \\
& & & & & \\
CP2K/SVP & - & -20.82 & - & - & - \\
CP2K/TZVPP & - & -3.46 & - & - & - \\
\hline \hline
\end{tabular}

Turning now to the BSSE in Table 5 we list its values for different basis sets and functionals. And again we observe here what has been known for a long time. The absolute value of the BSSE is largest for the smallest basis set, namely the SVP and the cc-pVDZ basis set. The Ahlrichs' basis sets reduce to a BSSE value of -2.4 to $-3.6 \mathrm{~kJ} / \mathrm{mol}$ depending on the particular functional whereas the Dunning basis set series reduces to -0.9 to $-1.4 \mathrm{~kJ} / \mathrm{mol}$. The TZVPPP basis set gives a higher absolute BSSE than the smaller TZVPP basis set. The TZVP basis set includes no $f$-function and one less $d$-function on the oxygen (see table 1 ) but more $s$-functions as opposed to the cc-pVTZ which results in an absolute value of the BSSE that is more than a factor two smaller than the BSSE absolute value of cc-pVTZ. For optimization with the SVP and TZVPP and the BLYP functional the CP2K values are also given in table 5. Single point calculations on the Turbomole geometries led to basically identical results (SVP: $-20.82 \mathrm{~kJ} / \mathrm{mol}$ and TZVPP: $-3.42 \mathrm{~kJ} / \mathrm{mol}$ ). The absolute BSSE of $\mathrm{CP} 2 \mathrm{~K}$ for the SVP basis set is reduced by a third compared to the TuRBomole value. Since a large absolute BSSE is not acceptable Krack and Parrinello introduced special designed GBSs where polarisation functions were added in order to further reduce this quantity. ${ }^{25}$ 
Table 6: Interaction energy $E_{I}$ in $\mathrm{kJ} / \mathrm{mol}$ for structure II. First block: Counterpoise corrected energies obtained with Gaussian basis set; Second block: Energies calculated with a plane wave basis set with $E_{\text {cut }}=150$ Ryd. The last two lines in the GBS block show values obtained with the GAPW code $\mathrm{CP} 2 \mathrm{~K}$

\begin{tabular}{|c|c|c|c|c|c|}
\hline \multirow[b]{2}{*}{ basis } & \multirow[b]{2}{*}{ BP86 } & \multicolumn{3}{|c|}{$\overline{E_{I}(\mathrm{GBS})}$} & \multirow[b]{2}{*}{ PBE0 } \\
\hline & & BLYP & B3LYP & $\overline{\mathrm{PBE}}$ & \\
\hline SVP & -17.16 & -10.59 & -18.46 & -15.33 & -21.24 \\
\hline TZVP & -21.25 & -20.81 & -22.40 & -24.50 & -24.24 \\
\hline TZVPP & -18.25 & -17.72 & -19.23 & -21.51 & -20.99 \\
\hline TZVPPP & -17.93 & -17.40 & -18.93 & -21.10 & -20.67 \\
\hline cc-pVDZ & -16.54 & -15.86 & -18.13 & -19.41 & -20.32 \\
\hline cc-pVTZ & -17.76 & -17.25 & -18.80 & -20.78 & -20.61 \\
\hline cc-pVQZ & -17.86 & -17.56 & -19.01 & -21.04 & -20.72 \\
\hline cc-pV5Z & -17.91 & -17.51 & -18.97 & -21.27 & -20.76 \\
\hline $\mathrm{CP} 2 \mathrm{~K} / \mathrm{SVP}$ & - & -15.85 & - & - & - \\
\hline \multirow[t]{2}{*}{$\mathrm{CP} 2 \mathrm{~K} / \mathrm{TZVPP}$} & - & -17.61 & - & - & - \\
\hline & & \multicolumn{3}{|c|}{$\overline{E_{I}(\mathrm{PWBS})}$} & \\
\hline$E_{\text {cut }}$ & BP86 & BLYP & B3LYP & $\mathrm{PBE}$ & PBE0 \\
\hline 150 & -18.40 & -17.44 & -18.84 & -21.66 & -21.23 \\
\hline
\end{tabular}

Table 6 contains the binding energies of conformation II. For reason of clarity we omitted interaction energies for the PWBS with an energy cutoff $E_{\text {cut }}$ between 20 Ryd and 110 Ryd. The interaction energies of structure II calculated with MP2/TZVPP and CCSD(T) in the basis set limit are $-19.2 \mathrm{~kJ} / \mathrm{mol}$ and $-20.7 \mathrm{~kJ} / \mathrm{mol}$ respectively. ${ }^{26}$ A recent and high-level correlated thus trustworthy value for the water dimer was provided by Klopper et al. with $-21.00 \mathrm{~kJ} / \mathrm{mol} .{ }^{27}$ We see that the PBE and PBE0 energies compare best with the CCSD(T) basis set limit values. BLYP provides values that least correspond to the CCSD(T) data. If we compare the stability of the water dimer given by the different functionals, all the PWBS calculations as well as GBS results show the same order. The intermolecular bond of the water dimer is strongest using the PBE functional, followed by PBE0, B3LYP, BP86, and BLYP. It is remarkable that the differences in binding energy between $E_{\text {cut }}=150$ Ryd PWBS calculations and the largest GBS calculations are less than $0.5 \mathrm{~kJ} / \mathrm{mol}$ for all functionals. Again we want to recall that for all functional the difference between 70 Ryd and 150 Ryd is less $(<1 \mathrm{~kJ} / \mathrm{mol})$ and so is the difference between the plane wave basis set results at 150 Ryd and the TZVPPP Gaussian basis set. Interestingly, the deviations provided by different functionals $(5 \mathrm{~kJ} / \mathrm{mol})$ and thus the difference to the benchmark value of Klopper and coworkers ${ }^{27}$ is much larger.

In the following tables we turn to the discussion of the geometry. We observe the usual 
functional trends, such as BLYP and B3LYP providing the largest intermolecular distances, see for example the TZVPPP row in table 7.

Table 7: Distance r1 and r2 in pm for conformation II for all functionals, basis sets and cutoff energies in Ryd. The GBS were abbreviated for clarity: TZV2P = TZVPP; TZV3P = TZVPPP; $\mathrm{C}=$ cc-p.

\begin{tabular}{|c|c|c|c|c|c|c|c|c|c|c|}
\hline \multirow[b]{2}{*}{ basis } & \multirow[b]{2}{*}{ BP86 } & \multicolumn{3}{|c|}{ r1 from GBS } & \multirow[b]{2}{*}{ PBE0 } & \multirow[b]{2}{*}{ BP86 } & \multicolumn{3}{|c|}{ r2 from GBS } & \multirow[b]{2}{*}{ PBE0 } \\
\hline & & BLYP & B3LYP & $\mathrm{PBE}$ & & & BLYP & B3LYP & $\overline{\mathrm{PBE}}$ & \\
\hline SVP & 188.9 & 192.6 & 190.6 & 207.2 & 188.7 & 98.6 & 98.6 & 97.5 & 98.2 & 97.2 \\
\hline TZVP & 190.5 & 194.6 & 193.0 & 190.0 & 190.1 & 98.2 & 98.2 & 97.1 & 98.1 & 96.8 \\
\hline TZV2P & 192.9 & 197.3 & 195.6 & 192.8 & 192.9 & 98.0 & 97.9 & 96.8 & 97.9 & 96.6 \\
\hline TZV3P & 193.3 & 197.9 & 195.9 & 192.8 & 193.1 & 97.9 & 97.8 & 96.8 & 97.9 & 96.6 \\
\hline CVDZ & 189.2 & 191.9 & 190.7 & 188.4 & 188.5 & 98.8 & 98.9 & 97.6 & 98.8 & 97.3 \\
\hline CVTZ & 191.4 & 195.5 & 194.4 & 190.7 & 191.5 & 98.1 & 98.0 & 96.9 & 98.0 & 96.6 \\
\hline CVQZ & 191.4 & 197.1 & 197.8 & 191.0 & 192.2 & 97.9 & 97.8 & 96.8 & 97.9 & 96.6 \\
\hline \multirow[t]{2}{*}{ CV5Z } & 191.9 & 196.9 & 197.8 & 191.6 & 193.5 & 97.9 & 97.8 & 96.8 & 97.9 & 96.5 \\
\hline & \multicolumn{4}{|c|}{ r1 from PWBS } & & \multicolumn{4}{|c|}{ r2 from PWBS } & \\
\hline$E_{\text {cut }}$ & BP86 & $\overline{B L Y P}$ & B3LYP & PBE & PBE0 & BP86 & $\overline{\mathrm{BLYP}}$ & $\overline{\text { B3LYP }}$ & $\mathrm{PBE}$ & PBE0 \\
\hline 20 & 213.4 & 215.1 & 215.5 & 213.3 & 251.1 & 116.8 & $\begin{array}{l}117.2 \\
\end{array}$ & 115.4 & 116.8 & 114.5 \\
\hline 50 & 193.2 & 208.9 & 191.3 & 199.8 & 194.4 & 98.2 & 98.7 & 97.8 & 99.0 & 97.7 \\
\hline 70 & 192.6 & 195.3 & 194.0 & 193.5 & 193.5 & 98.5 & 98.9 & 97.9 & 98.9 & 97.6 \\
\hline 90 & 193.8 & 196.0 & 193.6 & 191.2 & 194.4 & 98.4 & 98.2 & 97.3 & 98.2 & 97.0 \\
\hline 110 & 192.3 & 192.9 & 193.7 & 192.0 & 192.0 & 98.3 & 98.1 & 97.3 & 98.0 & 96.8 \\
\hline 150 & 194.1 & 191.7 & 195.0 & 192.0 & 193.5 & 98.2 & 98.1 & 97.1 & 98.0 & 96.8 \\
\hline
\end{tabular}

In general the PWBS geometries do not show trends like distances becoming shorter with larger basis set. However, the combinations of the GBSs and all functionals (with the exception of the SVP/PBE combination) show such trends. For example the r1 distance becomes larger with increasing basis set. We also note, that the differences in the BP86 r1 series are smallest. This is the reason that usually BP86 is preferred over other GGA functionals if reliable structures are sought for. Comparing now TZVPPP and cc-pV5Z with PWBS/150 we find that for all functionals the geometry values agree within $3 \mathrm{pm}$. Surprisingly the BLYP functional shows larger deviations of about $5 \mathrm{pm}$, see last row third column of table 7 and compare to TZVPPP and cc-pV5Z row also third column. 
Table 8: Distance r3 in pm and angle $\alpha$ in degrees of conformation II for all investigated electronic structure methods. Energy cutoff $\mathrm{E}_{\text {cut }}$ of the PWBS is given in Ryd. The GBS were abbreviated for clarity: TZV2P = TZVPP; TZV3P = TZVPPP; C = cc-p.

\begin{tabular}{|c|c|c|c|c|c|c|c|c|c|c|}
\hline \multirow[b]{2}{*}{ basis } & \multirow[b]{2}{*}{ BP86 } & \multicolumn{2}{|c|}{ re3 from GBS } & \multirow[b]{2}{*}{$\mathrm{PBE}$} & \multirow[b]{2}{*}{ PBE0 } & \multirow[b]{2}{*}{ BP86 } & \multicolumn{2}{|c|}{$\alpha$ from GBS } & \multirow[b]{2}{*}{ PBE } & \multirow[b]{2}{*}{ PBE0 } \\
\hline & & BLYP & B3LYP & & & & BLYP & B3LYP & & \\
\hline SVP & 286.2 & 289.3 & 286.9 & 274.1 & 285.2 & 7.4 & 9.2 & 7.4 & 39.0 & 5.6 \\
\hline TZVP & 288.5 & 292.3 & 289.9 & 287.6 & 286.8 & 2.9 & 4.2 & 2.6 & 4.9 & 2.1 \\
\hline TZV2P & 290.3 & 294.4 & 292.1 & 289.9 & 288.6 & 5.2 & 5.8 & 3.9 & 6.2 & 6.5 \\
\hline TZV3P & 290.7 & 295.2 & 292.3 & 289.8 & 289.1 & 4.4 & 5.0 & 4.5 & 6.0 & 4.8 \\
\hline CVDZ & 287.3 & 290.4 & 288.3 & 285.7 & 285.8 & 5.7 & 3.8 & 1.8 & 8.2 & 1.4 \\
\hline CVTZ & 289.3 & 293.3 & 291.2 & 288.5 & 288.0 & 2.5 & 3.0 & 2.7 & 2.7 & 3.2 \\
\hline CVQZ & 289.2 & 294.6 & 294.3 & 288.6 & 288.5 & 2.5 & 3.6 & 3.6 & 3.0 & 3.8 \\
\hline \multirow[t]{2}{*}{ CV5Z } & 289.6 & 294.5 & 294.3 & 289.2 & 289.6 & 3.0 & 3.7 & 3.6 & 3.8 & 4.1 \\
\hline & & \multicolumn{3}{|c|}{ r1 from PWBS } & & & \multicolumn{2}{|c|}{$\alpha$ from PWBS } & & \\
\hline$E_{\text {cut }}$ & BP86 & BLYP & $\bar{B} 3 \mathrm{LYP}$ & $\overline{\mathrm{PBE}}$ & PBE0 & BP86 & BLYP & $\overline{\text { B3LYP }}$ & PBE & PBE0 \\
\hline 20 & 330.0 & 332.2 & 330.7 & 329.9 & 329.5 & 2.1 & 1.8 & 2.1 & 2.5 & 2.5 \\
\hline 50 & 291.2 & 307.1 & 288.8 & 298.5 & 291.7 & 3.5 & 5.3 & 4.2 & 3.5 & 3.9 \\
\hline 70 & 290.8 & 293.9 & 291.6 & 292.1 & 290.8 & 3.5 & 3.6 & 3.8 & 3.5 & 3.8 \\
\hline 90 & 291.9 & 294.0 & 290.6 & 290.1 & 291.1 & 3.3 & 3.3 & 3.5 & 3.7 & 3.6 \\
\hline 110 & 290.3 & 290.7 & 290.7 & 289.7 & 287.0 & 3.6 & 3.7 & 3.6 & 3.7 & 4.1 \\
\hline 150 & 292.0 & 289.5 & 291.7 & 289.7 & 286.4 & 3.4 & 3.5 & 4.0 & 3.8 & 4.2 \\
\hline
\end{tabular}

In Table 8 distance $\mathrm{r} 3$ and angle $\alpha$ are given. The distance $\mathrm{r} 3$ behaves analogously to distance $\mathrm{r} 1$. The r3 distances of the GBSs compare well to the PWBS values for large basis sets. However, the very small PWBS (20 Ryd) leads to enlarged distances of about $40 \mathrm{pm}$. Agreement between PWBSs and GBSs starts with cutoff 70 Ryd. The angle varies for the GBS between 2 and 9 degree. There is one very large angle of 38 degree for the PBE/SVP combination. The plane wave basis set angles are a bit smaller ranging from 2 to 4 degrees. Again there is no convergence behavior. The difference between PWBSs and GBSs is mostly within $2^{\circ}$.

\section{References}

[1] Helgaker, T.; Gauss, J.; Jørgensen, P.; Olsen, J. J. Chem. Phys. 1997, 106, 6430.

[2] Bak, K. L.; Gauss, J.; Jørgensen, P.; Olsen, J.; Helgaker, T.; Stanton, J. F. J. Chem. Phys. 2001, 114, 6548.

[3] Pawlowski, F.; Halkier, A.; Jørgensen, P.; Bak, K. L.; Helgaker, T.; Klopper, W. J. Chem. Phys. 2003, 118, 2539. 
[4] Haynes, P. D.; Skylaris, C.-K.; Mostofi, A. A.; Payne, M. C. Chem. Phys. Lett. 2006, $422,345$.

[5] Hutter, J.; Curioni, A. ChemPhysChem 2005, 6, 1788.

[6] Allen, M. P.; Tildesley, D. J. Computer Simulation of Liquids; Clarendon Press: Oxford, 1987 reprinted 1990.

[7] Ahlrichs, R.; Bär, M.; Häser, M.; Horn, H.; Kölmel, C. Chem. Phys. Lett. 1989, 162, 165.

[8] "CPMD V3.8 Copyright IBM Corp 1990-2003, Copyright MPI für Festkörperforschung Stuttgart 1997-2001", see also www.cmpd.org (accessed May 1, 2007).

[9] http://cp2k.berlios.de/, page CP2k A General Program to Perform Molecular Dynamics Simulations (accessed May 1, 2007).

[10] Becke, A. D. Phys. Rev. A 1988, 38, 3098.

[11] Lee, C.; Yang, W.; Parr, R. G. Phys. Rev. B 1988, 37, 785.

[12] Perdew, J. P. Phys. Rev. B 1986, 33, 8822.

[13] Perdew, J. P.; Burke, K.; Ernzerhof, M. Phys. Rev. Lett. 1996, 77, 3865.

[14] Becke, A. D. J. Chem. Phys. 1993, 98, 5648.

[15] Stephens, P. J.; Devlin, F. J.; Chabalowski, C. F.; Frisch, M. J. J. Phys. Chem. 1994, 98, 11623.

[16] Adamo, C.; Barone, V. J. Chem. Phys. 1999, 110, 6158.

[17] Baerends, E. J.; Ellis, D. E.; Ros, P. Chem. Phys. 1973, 2, 41.

[18] Dunlap, B. I.; Connolly, J. W. D.; Sabin, J. R. Chem. Phys. 1979, 71, 3396.

[19] Todorova, T.; Seitsonen, A. P.; Hutter, J.; Kuo, I.-F. W.; Mundy, C. J. J. Phys. Chem. B 2006, 110, 3685.

[20] The turbomole basis set library is available via anonymous ftp from ftp://ftp.chemie.uni-karlsruhe.de/pub/basen.

[21] Dunning, T. H. J. Chem. Phys. 1989, 90, 1007.

[22] Spackman, M. A. J. Phys. Chem. 1989, 93, 7594.

[23] Martyna, G. J.; Tuckerman, M. E. Journal of Chemical Physics 1999, 110, 2810.

[24] Lee, H. S.; Tuckerman, M. E. J. Phys. Chem. A 2006, 110, 5549.

[25] Krack, M.; Parrinello, M. Phys. Chem. Chem. Phys. 2000, 2, 2105. 
[26] Kirchner, B. J. Chem. Phys. 2005, 123, 204116.

[27] Klopper, W.; van Duijneveldt- va de Rijdt, J. G. C. M.; van Duijneveldt, F. B. Phys. Chem. Chem. Phys. 2000, 2, 2227.

[28] Car, R.; Parrinello, M. Physical Review Letters 1985, 55, 2471. 